\title{
Future Biotechnology
}

\author{
Munis Dundar ${ }^{1}$, Satya Prakash ${ }^{2}$, Ratnesh Lal ${ }^{3}$ and Donald K. Martin ${ }^{4 \star}$
}

\begin{abstract}
The field of biotechnology is large and could be considered tritely as simply the development of technology that is based on biology. It is clear that the concepts of biotechnology can spread to cover many different fields of application and so the future developments in biotechnology will be similarly wide-ranging across many fields of applications. Here we focus onto medical biotechnology and further refine our discussion onto considering aspects of genetics and nanotechnologies that could impact on the development of future biotechnologies in the medical field. These areas that we consider in this brief article provide the basis for a panel discussion on Future Biotechnology at the European Biotechnology Congress held in Valencia, Spain in April 2019.
\end{abstract}

Keywords: Medical biotechnology, nanotechnology, genetics, future developments

'Department of Medical Genetics, Faculty of Medicine, Erciyes University, Kayseri, Turkey

${ }^{2}$ Biomedical Technology and Cell Therapy Research Laboratory, Department of Biomedical Engineering, Faculty of Medicine, McGill University, 3775 University Street, Montreal, Quebec H3A 2B4, Canada

${ }^{3}$ University of California San Diego, Jacobs School of Engineering, La Jolla, CA 920930403, U.S.A.

${ }^{4}$ University Grenoble Alpes, SyNaBi, TIMCIMAG/CNRS/INSERM, UMR 5525, F-38000, Grenoble, France

*Corresponding author: D. K. Martin E-mail: don.martin@univ-grenoble-alpes.fr

DOI: 10.2478/ebtj-2019-0006

(C) 2019 Authors. This work was licensed under the Creative Commons AttributionNonCommercial-NoDerivs 4.0 License.

\section{Introduction}

The field of biotechnology is large and could be considered tritely as simply the development of technology that is based on biology. For example, the long history of utilizing the biological processes of microorganisms to produce bread or cheese. Nonetheless, modern biotechnology provides breakthrough products and technologies to fight debilitating and rare diseases, to reduce people's environmental footprint, to provide solutions for enhancing the world's food supply; to use less and cleaner sources of energy, and to have safer, cleaner and more efficient industrial manufacturing processes (1). It is clear that concepts of biotechnology can spread to cover many different fields of application and so the future developments in biotechnology will be similarly wide-ranging across many fields of applications. Here we focus onto medical biotechnology and further refine our discussion onto considering aspects of genetics and nanotechnologies that could impact on the development of future biotechnologies in the medical field.

\section{Recent Developments in Genetics}

The Human Genome Project was initiated in 1990 and was completed in 2003, with the joint efforts of scientists in many countries, public programs and private companies in order to expose all genes in the human genome (2). An important outcome of the Human Genome Project was that electronically compiled genomic data could be used to identify desired genes in certain parts of the genome (Fig.1). By this time, DNA repeat sequences were considered insignificant but were thought to change or rearrange the genome throughout the evolutionary process. At the same time, learning the role of these sequences would help solve the structure and dynamics of the chromosomes. Despite the enormous information that is difficult to fit into standard computers, many questions remain unanswered and a complete map of human DNA cannot be created (3). 


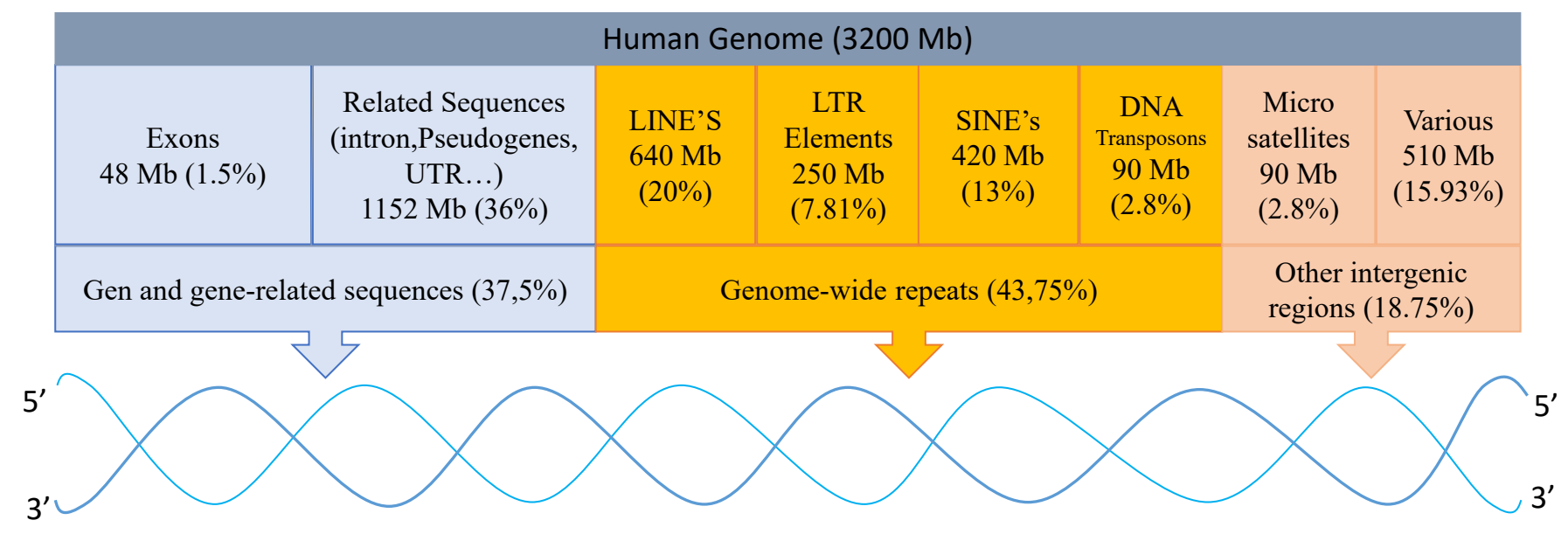

Figure 1. Overview of the human genome. Gene and gene-related sequences cover $37.5 \%$ of the human genome, while genome-wide repeats constitute $43.75 \%$. The remaining $18.75 \%$ are intergenic regions.

Pharmacogenetic science is being developed with more and more comprehensive information obtained from the Human Genome Project. The response of a patient to chemotherapeutic agents given for cancer can vary with hereditary genetic variations. Pharmacogenetics is intended to use information about this diversity to plan the treatment with reduced toxicity and better response. Previous studies have mostly focussed on single nucleotide polymorphisms, but more broadly it is important to consider genetic diversity in all biological and pharmacological pathways (4). In Europe, the disease prevention system and personalised health care based on a detailed examination of the patient are in the forefront. In this context, it is necessary to create a vision in health services by focussing on information and communication technologies and especially preventive health services. The disease prevention system is primarily based on a detailed description of the individual (e.g. clinical, molecular, imaging and sensor-based). The idea of personalised medicine consists of genomic risk assessment along with other clinical trials, and the personalised medicine and personal genomic language is now in common use. Future personalised biosensors can perform measurements that transmit clinical passwords and blood biomarkers to a cluster in real time or send alerts when they are in the earliest stages of a stroke, for example. Drugs that are specially prepared or produced by $3 \mathrm{D}$ printing based on pharmacogenomic data are also innovations in this context.

\section{Genetic Engineering}

Another developing area in this sense is genetic engineering. Genetic engineering can potentially regulate hereditary diseases. Brain-computer interfaces developed by using new nanomaterials that provide bidirectional neural communication will enable the patients to write words which they are imagine or to control the devices. These non-invasive interfaces may seem utopian, but can be a marvel of genetic engineering in the new era. In addition, studies are being carried out on treatments that may extend life, such as telomere modifications and reversing aging. In modern medicine, doctors evaluate patients to clinically diagnose, treat, and prevent diseases. Thus, there are also benefits of a treatment regime that is based on the genotype of each individual to include epigenetic factors for the development of individualised medication selection, dose adjustment and individualised therapies to overcome a more traditional trail-and-error approach. For example, in the field of oncology, which plays a role in the prevention, management and treatment of non-future cancers, there have been enormous strides that can be attributed to the development of immunotherapy, genomic and genetic engineering technology (5).

The other new face of modern genetic engineering is CRISPR / Cas9 technology and its therapeutic potential is excellent. As technology develops, the therapeutic potential of CRISPR / Cas9 will continue to increase. Nonetheless, CRISPR / Cas9 has many difficulties in fully developing its potential. Cas9 nickases and mutants that reduce non-specific DNA binding are designed to alleviate these problems, although this is a problematic solution. The gene cargo distribution system remains the biggest obstacle to the routine use of CRISPR / Cas9, and a multipurpose delivery method has not yet emerged (6). Gene therapy is an experimental method to correct a defective gene that is responsible for the emergence of the disease. In addition to advances in delivery and expression technologies, future efforts will focus on new areas of gene therapy practice, such as new resistance genes and chimeric T-cell receptors. The sequence of the human genome is useful in many fields, from molecular medicine to human evolution. With the development of gene therapy technologies, the modelling of genetic diseases also increased (7). In recent years, the renowned method of gene therapy has been used to treat a number of diseases on genetic models developed by CRISPR technology. For example, metabolic diseases, cardiovascular diseases, monogenic diseases. Also developed drugs are tested on these genetic models. If the correct gene is determined and the appropriate vectors are selected, then there should not be disease that cannot be treated. 


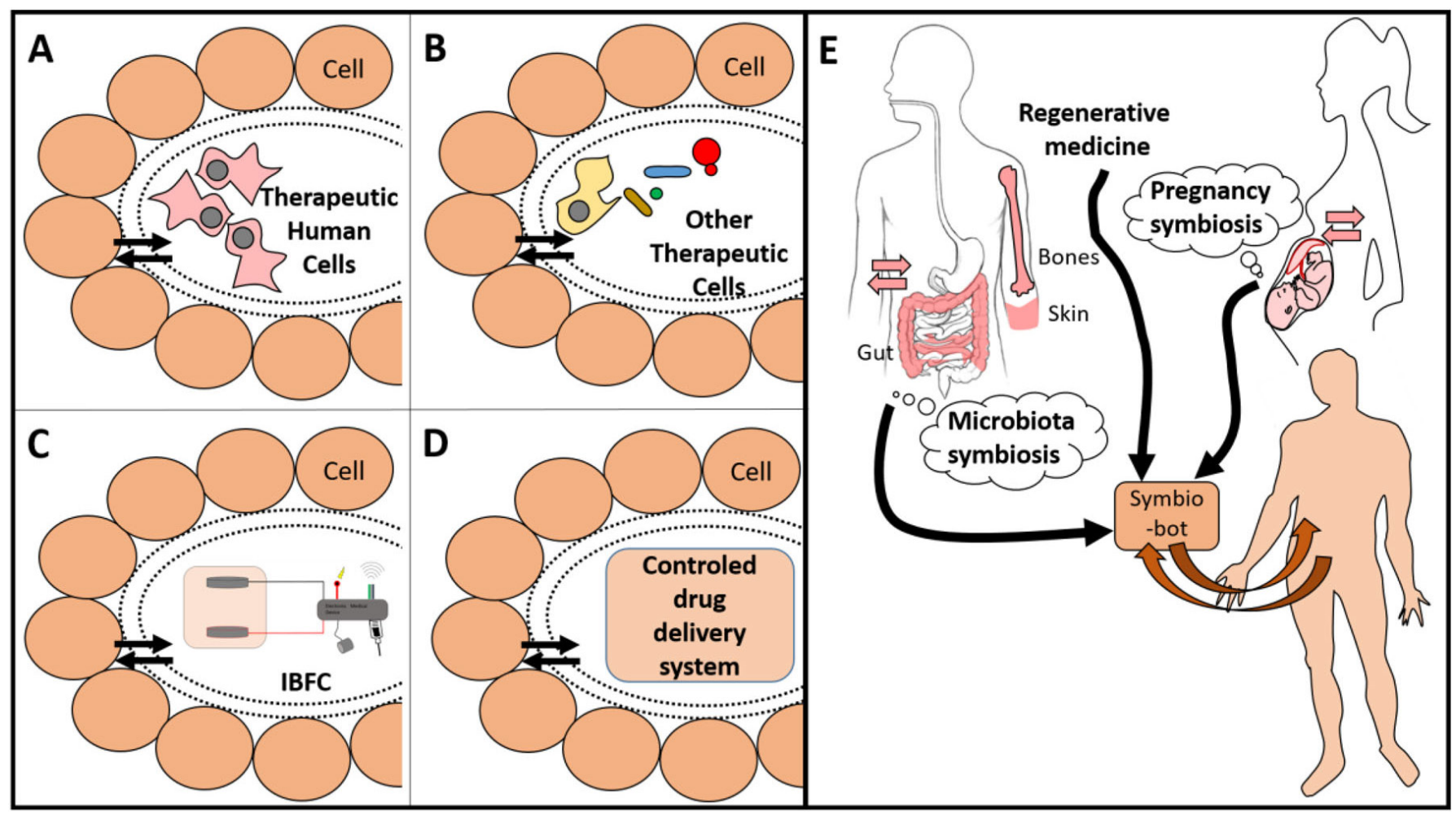

Figure 2. Examples of symbio-bots (A-D) that can be created in a bioinspired way (E). Each device is separated with a smart porous packaging that allows a duplex communication. Therapeutic cells ( $A$ and $B$ ) need a porous encapsulation that avoids an immune reaction and allows protection from both sides. They may be human cells, as MSC or specialized cells such as $\beta$-cell from Langherans islets (A), or other eukaryotic or prokaryotic cells (B). Panel C shows an IBFC linked to an electronic medical device. Panel $\mathrm{D}$ shows a generic device delivering a therapeutic molecule. Panel E: Existing symbiosis (i.e., microbiota or pregnancy) are a source of bio-inspiration to establish a duplex communication between the body and its implants. Regenerative medicine should embrace this concept of bioinspiration for a better design and integration of implants, especially for future symbio-bots. (reproduced with permission from Alcaraz et al (10)).

\section{Nanotechnology and Medical Biotechnology}

Advances in nanotechnology also provide a rich framework for future developments in medical biotechnology. Related to disease prevention is the widespread use of antibiotics and antibacterial coatings to reduce the impact of infection on causing the failure of implanted medical devices. Nonetheless, such approaches have limited effectiveness. Nanotechnology now provides the tools for nano-texturing the surfaces of materials for medical implants, with the aim to mimic the bactericidal properties of some animal, plant and insect species, and their topographical features. For example, the surface nano-structures of cicada, dragonfly and butterfly wings, shark skin, gecko feet, taro and lotus leaves provide self-cleaning and bactericidal properties (8). That type of bioinspiration provides great innovations in providing some biological-like characteristics that can be used to guide the surface structuration and synthesis of materials into functional devices and processes. However, there are potentially more benefits to medical biotechnology than simply using biology as a template to guide the surface structuration and synthesis of materials.

In biological systems there is a self-assembly of molecules to create elegant nanostructured systems. A central feature of such biological nanostructured systems is the assembly of phospholipid bilayer membranes that both provide compartments (i.e. biological cells) to rationalise the overall function of complex organisms (e.g. plants, animals) and also to provide an environment in which to stabilise membrane proteins to assist in the sensing and actuating functions of biological cells (9). These components of biological cells provide the basis for transport of ions and molecules between the cell and the surrounding environment inside the body, which supports the ability of cells to participate in physiological control of the body. Extending the concept of bioinspiration to include such elegant nanostructured biological self-assembly then provides an additional dimension to applying nanotechnology to medical biotechnology. That additional dimension is to include nanostructured bio-membranes into hybrid medical devices that can provide the ability for the medical device to communicate ions and molecules with the body. This then leads to the notion that an implanted medical device should integrate and become symbiotic with the body (Fig. 2). This extends the definition of a biocompatible system to one that requires stable exchange of materials between the implanted device and the body. Having this novel concept in mind will guide research in a new field between medical implant and regenerative medicine to create actual symbiotic devices (10). 


\section{Impacts on Novel Biotechnologies}

Additionally, a treatment method in the future of medicine is the digestible sensors placed in pills. Data from these sensors are transmitted to doctors and family. Thanks to the biotechnology industry, more objects can now be printed using 3D printers. In the near future, printing of medical devices in underdeveloped areas and printing of live tissues, cells or drugs may be imagined. But, everyone is able to print medicines containing patented molecules in their homes, so ethical problems can emerge. Because the growing world of the pharmaceutical industry and biotechnology creates the difficulty here.

Also $3 \mathrm{D}$ printing is not the only way used to create body parts and artificial organs, it can be grown in the laboratory environment using biomaterials. The artificial organ is a device or biological material that is implanted in the body to alter a natural organ or function. The other innovation can be the sensors that can be digested to make a quick diagnosis. These sensors can be swallowed directly for gastrointestinal diseases. Further, the sensors embedded in the tooth can detect jaw movements and speech. More complex microchips that can mimic the whole human body are needed, and this final solution could arrive soon. Item, as the amount of information increases, $\operatorname{cog}$ nitive computers are used instead of human in medical decision making.

\section{Concluding Remarks}

Synthetic biology can provide useful technologies, such as constructing living things from simple and well-characterized parts, microbial fuel factories, immune cells designed to selectively attack cancer, live tissues and new materials. The complete composition of the global minimal genome is still unknown, and the role of the medium in determining the cell type or condition-specific underlying genes is also vague. In the last 20 years, fundamental technologies for minimal genome construction have been developed, and in the future researchers will build ideal stable hosts using the least genome for scientific and industrial aims (11). Genetic circuits can be easily designed by integrating DNA into a minimal genome. This will accelerate the construction of customized industrial genomes for the biologic recovery of environmental toxins, the manufacture of beneficial drugs and chemicals, or the creation of renewable energy sources.

The first FDA's Orphan Drug Act was offered by US in 1983. After the US, in 2000 Singapore, Japan, Australia became the other countries. Also, European Union was presented their own shape of orphan medicine. Comparative studies provided very positive effect on market. Before the act only about 10 orphan-like product was known but now the number excess of 230. Similarly, this number in Japan reach to 175 . But be- fore this number was only 42 . Biotechnology is introducing an ever-increasing role in drug discovery. Because it prefers new approaches for the development of drugs. With the advance of orphan drug legislation these attempts are offering new drugs for patients. Moreover, the ratio about $50 \%$ of orphan drugs are biotechnology based. For example, in Europe, Since the advance of biotech-based orphan drugs development time for new biotech-based drugs is longer than for reformulation of existing classical drugs for orphan indications, this ratio is about $35-40 \%$ but increasing (12).

\section{Acknowledgement}

This article provides a basis for the panel discussion on Future Biotechnologies, which is a scheduled Plenary Session in The European Biotechnology Congress held in Valencia, Spain from 11-13 April 2019.

\section{Conflict of interest statement}

The authors declare no financial or commercial conflict of interest.

\section{References}

1. Biotechnology Industry Organisation, https://www.bio.org/ what-biotechnology

2. Moraes F, Goes A. A decade of human genome project conclusion: scientific diffusion about our genome knowledge. Biochemistry and Molecular Biology Education 2016; 44(3): 215223.

3. Wilson BJ, Nicholls SG. The Human genome project, and recent advances in personalized genomics. Risk Management and Healthcare Policy; 2015; 8: 9-20.

4. Ulrich CM, Robien K, McLeod HL. Cancer pharmacogenetıcs: polymorphısms, pathways and beyond, Nature Reviews Cancer; 3(12): 812-920.

5. Wang $F$. The once and future of medicine. Science Insights 2018; 2018:e000051.

6. Lino CA, Harper JC, Carney JP, Timlin JA. Delivering CRISPR: a review of the challenges and approaches. Drug Delıvery 2018; 25(1): 1234-1257.

7. Kulkarni KM, Khot AM, Lokapure SG, Jadhav S. A brief review on gene therapy, Indo American Journal of Pharmaceutical Sciences 2018; 5(5): 3288-3299.

8. Jaggessar A, Shahali H, Mathew A, Yarlagadda PKDV. Bio-mimicking nano and micro-structured surface fabrication for antibacterial properties in medical implants. Journal of Nanobiotechnology 2017; 15(1):64.

9. Bentley WE, Martin DK, Gotoh T. Biomimetic and bioinspired biotechnology. Biotechnology Journal 2018; 13:1800670

10. Alcaraz JP, Cinquin P, Martin DK. Tackling the concept of symbiotic implantable medical devices with nanobiotechnologies. Biotechnology Journal 2018; 13:1800102.

11. Coyle M, Hu J,Gartner Z. Mysteries in a minimal genome, ACS Central Science 2016; 2(5): 274-277.

12. Tambuyzer E. Biotechnology-based orphan drugs achievements and challenges. Drug Discovery World 2003; Fall: 59-65. 